Supporting Information

\title{
Purification, conformational analysis, and properties of a family of tigerinin peptides from skin secretions of the crowned bullfrog Hoplobatrachus occipitalis (Dicroglossidae)
}

Christopher M. McLaughlin, $† \#$ Sandrina Lampis, $\neq \#$ Milena Mechkarska, $\nmid+\infty$ Laurent Coquet, § Thierry Jouenne, § Jay D. King, $\perp$ Maria Luisa Mangoni, \|, Miodrag L. Lukic, $\mathbf{\nabla}$ Mariano A. Scorciapino, $\neq$ J. Michael Conlon $\dagger^{*}$

'SAAD Centre for Pharmacy and Diabetes, School of Biomedical Sciences, University of Ulster, Coleraine, U.K.

${ }^{*}$ Department of Chemical and Geological Sciences and Department of Biomedical Sciences - Biochemistry Unit, University of Cagliari, Italy

${ }^{\S}$ CNRS UMR 6270, PISSARO, University of Rouen, Institute for Research and Innovation in Biomedicine (IRIB), Mont-Saint-Aignan, France

${ }^{\perp}$ Rare Species Conservatory Foundation, St. Louis, MO, USA

| Instituto Pasteur-Fondazione Cenci Bolognetti, Department of Biochemical Sciences, Sapienza University of Rome, Rome, Italy

$\nabla$ Center for Molecular Medicine, Faculty of Medicine, University of Kragujevac, Kragujevac, Serbia 

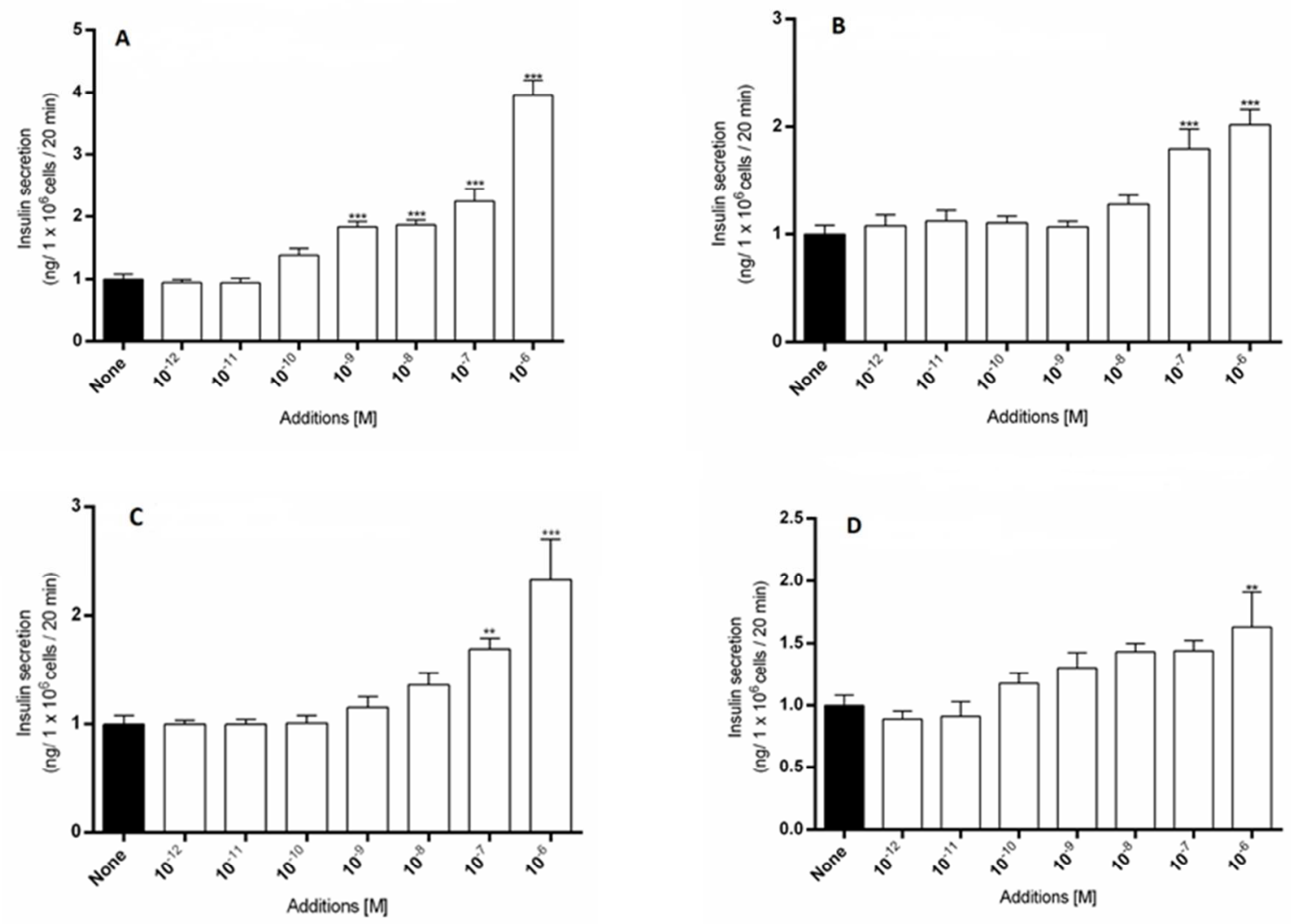

Figure S1. Effect of synthetic (A) tigerinin-1O, (B) tigerinin-2O, (C) tigerinin-3O and (D) tigerinin-4O on the rate of release of insulin from BRIN BD-11 clonal $\beta$-cells, Values show mean $\pm \operatorname{SEM}(\mathrm{n}=6) . * * * \mathrm{P}<0.001, * * \mathrm{P}<0.01, * \mathrm{P}<0.05$ compared to $5.6 \mathrm{mM}$ glucose alone. Data showing the effect of established stimulatory agents on the rate of release of insulin are provided in the text. 

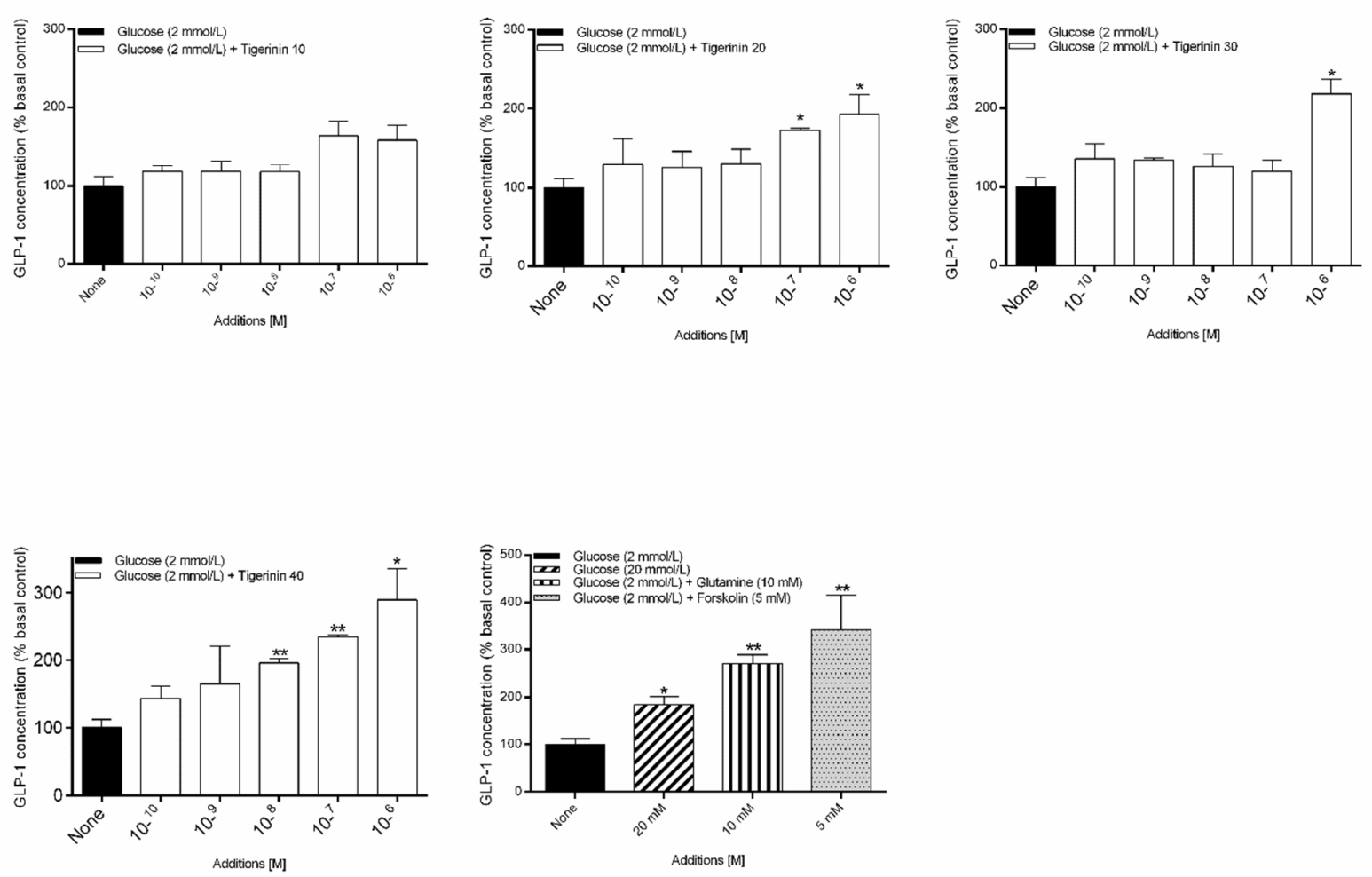

Figure S2. Effect of synthetic tigerinin-1O,-2O, $-3 \mathrm{O}$ and $-4 \mathrm{O}$ and established stimulatory agents on the rate of release of GLP-1 from GLUTag murine enteroendocrine cells, Values show mean $\pm \operatorname{SEM}(\mathrm{n}=3) . * * \mathrm{P}<0.01, * \mathrm{P}<0.05$ compared to $5.6 \mathrm{mM}$ glucose alone. 

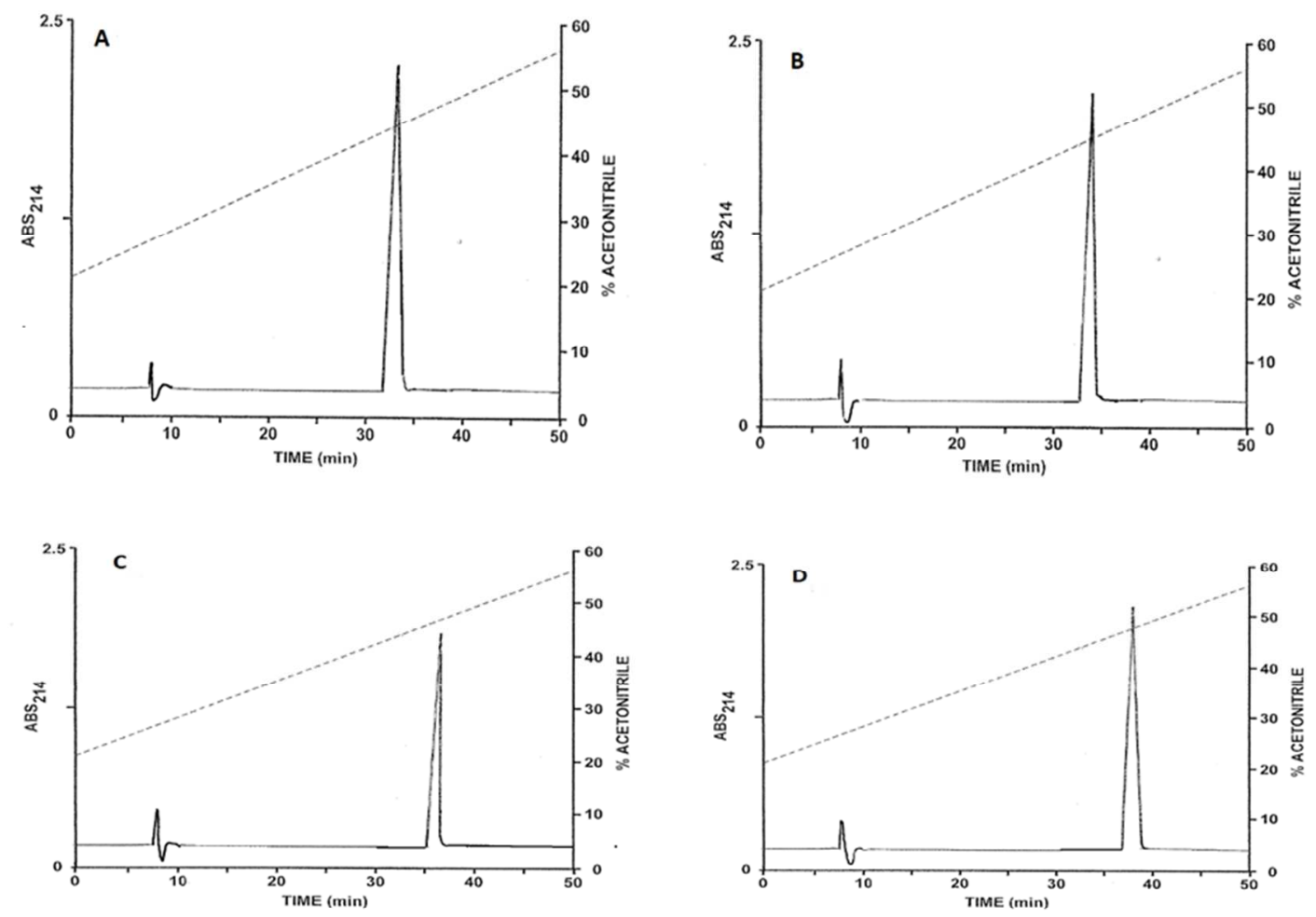

\section{Figure S3.}

Elution profiles on a semipreparative Vydac C-8 column of synthetic (A) tigerinin-1O, (B) tigerinin-2O, (C) tigerinin-3O, and (d) tigerinin-4O after initial purifications on preparative Vydac C-18 and semipreparative Vydac C-4 columns. The data indicate that the peptides used for biological testing were $>98 \%$ pure. 


\begin{tabular}{|c|c|c|c|c|c|c|}
\hline & \multicolumn{4}{|l|}{${ }^{1} \mathbf{H}$} & \multicolumn{2}{|l|}{${ }^{13} \mathrm{C}$} \\
\hline & HN & $\mathbf{H} \alpha$ & $\mathbf{H} \boldsymbol{\beta}$ & Others & $\mathrm{C} \alpha$ & $C \beta$ \\
\hline \begin{tabular}{|l|} 
R1 \\
\end{tabular} & - & 4.15 & 1.99 & $\begin{array}{|lll|}\gamma & 1.66 \\
\delta & 3.23 \\
\varepsilon & 7.24 \\
\end{array}$ & 55.69 & 27.64 \\
\hline \begin{tabular}{|l|} 
T2 \\
\end{tabular} & 8.75 & 4.37 & 4.08 & $\gamma 1.23$ & 62.48 & 70.07 \\
\hline \begin{tabular}{|l|} 
C3 \\
\end{tabular} & 8.62 & 4.69 & 3.08 & & 56.09 & 41.18 \\
\hline I4 & 8.42 & 4.52 & 1.88 & 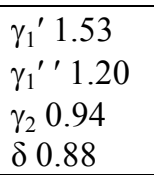 & 58.72 & 38.96 \\
\hline P5 & - & 4.24 & $\begin{array}{l}2.31 \\
2.11\end{array}$ & $\begin{array}{ll}\gamma^{\prime} & 1.98 \\
\gamma^{\prime} & 1.86 \\
\delta^{\prime} & 3.82 \\
\delta^{\prime \prime} & 3.66 \\
\end{array}$ & 65.39 & 31.77 \\
\hline \begin{tabular}{|l|} 
I6 \\
\end{tabular} & 8.30 & 3.98 & 1.94 & $\begin{array}{lll}\gamma_{1}^{\prime} & 1.66 \\
\gamma_{1}^{\prime \prime} & 1.53 \\
\gamma_{2} & 0.92 \\
\delta & 0.89\end{array}$ & 57.23 & 40.11 \\
\hline \begin{tabular}{|l|} 
P7 \\
\end{tabular} & - & \begin{tabular}{|l|l|}
4.49 \\
\end{tabular} & $\begin{array}{l}2.11 \\
2.05\end{array}$ & $\begin{array}{|ll|}\gamma & 1.99 \\
\delta^{\prime} & 3.86 \\
\delta^{\prime} & 3.74 \\
\end{array}$ & 63.85 & 33.15 \\
\hline \begin{tabular}{|l|} 
L8 \\
\end{tabular} & 8.00 & 4.61 & 1.95 & $\delta 0.97$ & 59.17 & 31.08 \\
\hline \begin{tabular}{|l|} 
V9 \\
\end{tabular} & 7.90 & 4.20 & 2.08 & $\begin{array}{lll}\gamma_{1} & 0.92 \\
\gamma_{2} & 0.88\end{array}$ & 62.43 & 33.76 \\
\hline \begin{tabular}{|l|} 
M10 \\
\end{tabular} & 8.45 & 4.57 & $\begin{array}{l}2.13 \\
2.03 \\
\end{array}$ & $\gamma 2.66$ & 55.35 & 31.47 \\
\hline C11 & 8.15 & \begin{tabular}{|l|l}
4.47 \\
\end{tabular} & $\begin{array}{l}2.98 \\
3.20 \\
\end{array}$ & & 56.78 & 42.25 \\
\hline
\end{tabular}

